Website: http://revistas.lamolina.edu.pe/index.php/acu/index

(C) Universidad Nacional Agraria La Molina, Lima - Perú

\title{
Condición corporal y su relación con la producción de leche y el número de servicios por preñez en vacas Holstein
}

\section{Corporal condition and its relationship with milk production and pregnancy in Holstein cows}

\author{
Diana Jennifer Castro Alvarez ${ }^{1}$; María Elisa Catalina García Salas²*; Germán Rodríguez Franco ${ }^{3}$; Ericson Ruiz Figueroa² \\ * Autor de correspondencia
}

\section{Resumen}

El objetivo del presente estudio fue identificar y cuantificar el efecto de la condición corporal al momento de la seca (BCSSECA), parto (BCSPARTO), primer servicio post-parto (BCS1SERV) y concepción (BCSCONCEP) sobre la producción de leche a los 305 días (P305) y el número de servicios por preñez(NSERV) en vacas Holstein, de un establo en Puente Piedra en Lima. Los datos provienen de vacas que registraron por lo menos un parto en el periodo: 20102013, con lactancias terminadas, partos normales y periodos post-parto libre de enfermedades.Se empleó un modelo de regresión lineal múltiple encontrando que las variables independientes BCSSECA tuvieron efectos significativos ( $\mathrm{p}<$ $0,01)$ sobre la producción de leche a los 305 días; por cada punto de condición corporal que gane el animal por encima del ideal $(3,0$ a 3,5) al momento del secado, se pierde aproximadamente 106 litros de leche. El promedio de condición corporal fue 2,74; 2,90; 2,51 y 2,54 para BCSSECA, BCSPARTO, BCS1SERV y BCSCONCEP respectivamente. Se analizó el número de servicios por preñez con un modelo de regresión logístico, se ajustó el resultado de cada servicio en función de su éxito (vaca preñada $=1$, vaca vacía $=0$ ), la condición corporal no influyó sobre el resultado del servicio. Sin embargo, la condición corporal en el momento de secado, el número de lactancia y la edad al parto influyeron significativamente $(\mathrm{p}<0,01)$ sobre la producción de leche a los 305 días, de incrementarse un punto de condición corporal durante el secado, por encima del ideal $(3,0$ a 3,5) este impactaría negativamente sobre la producción de leche.

Palabras clave: condición corporal; número de servicios; producción de leche; reproducción; holstein.

\begin{abstract}
Abstrac
The objective of the present study was to identify and quantify the effect of body condition at dry period (BCSSECA), calving (BCSPARTO), first postpartum service (BCS1SERV) and conception (BCSCONCEP) on milk production at 305 Days (P305) and the number of pregnancy services (NSERV) in Holstein cows from a stable in Puente Piedra at Lima. The data come from cows that registered at least one calving in the period: 2010 - 2013, with finished lactations, normal parturitions and postpartum periods free of disease. A multiple linear regression model was used to find that the independent variables BCSSECA and age at calving (EPARTO) had significant effects $(\mathrm{p}<0.01)$ on milk production at 305 days; For each point of body condition that the animal gains above the ideal $(3,0$ to 3,5$)$ at the time of drying, approximately 106 liters of milk is lost. The average body condition was 2,74; 2,90; 2,51 and 2,54 for BCSSECA, BCSPARTO, BCS1SERV and BCSCONCEP respectively. The number of pregnancy services with a logistic regression model was analyzed, the result of each service was adjusted according to its success (pregnant cow $=1$, empty cow $=0$ ), the body condition did not influence the result of the service.
\end{abstract}

Keywords: body condition; number of services; milk production; reproduction; holstein.

\section{Introducción}

La condición corporal (ECC), es una herramienta útil y práctica para evaluar visualmente y al tacto el estado nutricional del ganado (García, 2008); es un indicador de la cantidad de reservas energéticas almacenadas en vacas lecheras (Grigera y Bargo, 2005), cuando el suministro de energía es mayor que la demanda, el exceso es almacenado en forma de grasa corporal, la misma que esposteriormente movilizada en épocas de escasez, y al que recurre el propio organismo como fuente de energía interna cuando los componentes calóricos de la ración absorbidos en el tracto digestivo son insuficientes (López, 2006).

La evaluación de la condición corporal al secado es útil para corroborar que la alimentación durante los últimos meses de lactancia haya permitido una correcta recuperación de reservas corporales, durante este periodo, los animales no deberían perder condición corporal (Grigera y Bargo, 2005).

Durante las primeras cuatro a seis semanas post-parto, el consumo de alimento de la vaca no aumenta tan rápido

\footnotetext{
${ }^{1}$ Ing. Zootecnista Práctica privada.

${ }^{2}$ Facultad de Zootecnia, Universidad Nacional Agraria La Molina, Lima, Perú. Email: megarcia@lamolina.edu.pe

${ }^{3}$ Facultad de Zootecnia, Universidad Nacional Agraria La Molina, Lima, Perú, E-mail: grodriguez@lamolina.edu.pe
} 
como la producción de leche, lo que resulta en movilización de las reservas corporales, por lo tanto, durante los primeros dos meses de lactancia, el grado al que una vaca va a perder condición corporal es determinado por el balance entre su capacidad de captación de nutrientes y su potencial genético para producción de leche (García, 2008).Se recomienda que las vacas al momento del parto deben alcanzar una condición corporal de 3,0 a 3,5 puntos (Hutjens, 2007).

Las vacas con baja condición corporal (1,5 en 1-5 escala) al parto tienden a perder menos puntos de condición corporal, al incrementar en menor tiempo su consumo de materia seca y esto les permite mantener e incrementar la producción, en cambio en los animales con BCS alta $(>3,0)$, pierden más rápidamente condición corporal, presentan anestros más prolongados y alguna enfermedad metabólica, demostraron Frood and Croxton (1978) citados por Montiel (2005).

El rápido incremento en los requerimientos de lactación causa un balance energético negativo durante el postparto temprano que puede prolongarse varias semanas. La repartición de nutrientes y el balance energético negativo afectan la concentración de hormonas que controlan la función reproductiva (Lucy, 2001).

La leche, la lipólisis proporciona un sustrato de energía para los tejidos no mamarios en los primeros días de la lactancia, evitando de este modo un mayor empleo de la glucosa para la síntesis de la lactosa por la glándula mamaria y el aumento de la producción de leche, según Bauman citado por Roche (2009).

El objetivo del presente estudio fue identificar y cuantificar el efecto de la condición corporal al momento de la seca (BCSSECA), parto (BCSPARTO), primer servicio post-parto (BCS1SERV) y concepción (BCSCONCEP) sobre la producción de leche a los 305 días (P305) y el número de servicios por preñez (NSERV) de vacas Holstein.

\section{Materiales y métodos}

El estudio se realizó en el establo Monte Grande, ubicado en el departamento de Lima, provincia de Lima, distrito de Puente Piedra; sus coordenadas geográficas son $11^{\circ} 51^{\prime} 30^{\prime}$ de latitud sur y $77^{\circ} 05^{\prime} 25^{\prime}$ " de longitud oeste; a una altitud de 190 m.s.n.m.(Collantes et al., 1999), está ubicado geográficamente en la Zona Baja del Valle del Río Chillón que se caracteriza por presentar un clima extremadamente árido; esta zona presenta una temperatura promedio anual de $20^{\circ} \mathrm{C}$, con un mínimo de $12^{\circ} \mathrm{C}$ en invierno y un máximo de $30{ }^{\circ} \mathrm{C}$ en verano. La humedad relativa promedio anual es del 77,6\%, llegando hasta el $95 \%$ en época de invierno.

Los animales son vacas Holstein puras, debidamente registradas en la Asociación Holstein del Perú y se crian bajo un sistema estabulado. El establo cuenta con dos salas de ordeño tipo espina de pescado de doble manga, una ubicada en la zona de vacas adultas y la otra en la zona de recría donde están las vacas de primer y segundo parto. La alimentación de las vaquillas, vaquillonas, vacas secas 474 y vacas en producción es en base a concentrado y forraje.

Las vacas se inseminan a partir de 60 días después del parto, teniendo que presentar flujo limpio y como mínimo 2,5 de condición corporal, después de ello examinar con el espéculo la condición de la cérvix y el flujo. El ordeño se realiza dos veces al día; la primera a las dos de la mañana y la última a las dos de la tarde; cada ordeño dura aproximadamente cuatro horas.

Se emplearon los Registros existentes en el establo, que contienen la información productiva, proporcionada por el Servicio Oficial de Productividad Lechera (SOPL) de la Cuenca de Lima, del Programa de Mejoramiento Animal de la Universidad Nacional Agraria La Molina, estos nos brindaron la siguiente información: código de la vaca (VACA), fecha de parto (FPARTO), número de parto (NPARTO), fecha de secado (FSECA), y producción de leche a los 305 días (P305).

De la base de datos del Software Agritec 8.5.0 de la Edición VaquitecEnterprise 2012 que contiene información productiva y reproductiva, se recolectó la fecha de servicio-concepción (FCONCEP), fecha de parto (FPARTO), números de parto (NPARTO), edad al parto (EPARTO), días vacíos (DVACIO), intervalo entre partos (INTERPARTO). A los cuales se les incorporó la condición corporal al momento del secado (SCSSECA), primer servicio post-parto (BCS1SERV), servicio-concepción (SCSCONCEP) y parto (SCSPARTO).

Se recopiló datos de los registros existentes en el establo, se consideró campañas completas de los animales que hayan tenido por lo menos un parto entre los años del 2010 al 2013. Se usaron 346 lactaciones de un total de 99 animales. Las calificaciones de condición corporal de acuerdo a los eventos de seca, parto y servicio-concepción fueron incorporados a partir de relación entre las fechas de parto, seca y concepción. El establo Monte Grande trabaja el tema de calificación corporal en números enteros y medios puntos a partir del año 2009.

Para la evaluación de la condición corporal de las vaquillas seutilizó el sistema de evaluación americano,se inició con las hembras que alcanzaron el peso y la edad óptima para el servicio, siendo este de $360 \mathrm{~kg}$ y 12 meses de edad, respectivamente Para estimar el peso se empleó una cinta torácica para vacas de la raza Holstein, esta rodea el tórax de la ternera y de acuerdo a su longitud nos permite determinar el peso aproximado; a la par se determina su condición corporal a través de una evaluación visual y palpación de los depósitos de grasa en la tuberosidad isquiática, apófisis transversas, vértebras espinosas; como también por la profundidad de las cavidades del ijar y la base la cola. En el caso de vacas multíparas se determinó la condición corporal en el momento del secado, parto y servicio, utilizándose el sistema de evaluación israelí.

Para evaluar el efecto de la condición corporal sobre la producción de leche a los 305 días, se empleó el modelo de regresión lineal múltiple, el cual explica la relación entre la variable dependiente: P305 y las variables independientes: BCSSECA, BCSPARTO, BCS1SERV, BCSCONCEP, ESTPARTO y EPARTO la cual se expresa 
mediante la siguiente ecuación: P305 $=\mathrm{b}_{0}+\mathrm{b}_{1}$ BCSSECA $+b_{2}$ BCSISERV + $b_{3}$ BCSCONCEP + ${ }_{4}$ BCSPARTO + $\mathrm{b}_{5}$ NLACTANCIA $+\mathrm{b}_{6}$ ESTPARTO $+\mathrm{b}_{7}$ EPARTO $+\varepsilon$.

Donde: P305 (Producción de leche a los 305 días), $\mathrm{B}_{0}$ (Intercepto), BCSSECA (Valores de la condición corporal de la vaca en el momento de seca), BCS1SERV (Valores de la condición corporal de la vaca al primer servicio postparto), BCSCONCEPT (Valores de la condición corporal de la vaca al quedar preñada), BCSPARTO (Valores de la condición corporal de la vaca al momento del parto), NLACTANCIA ( $\mathrm{N}^{\circ}$ de lactancia), ESTPARTO (Estación al momento del parto en invierno y/o verano), EPARTO (Edad de la vaca al momento del parto en meses).

Para evaluar el efecto de la condición corporal sobre el parámetro reproductivo: número de servicios por preñez, se empleó el modelo de regresión logística. Este modelo, indica que tan probable es que una vaca quede preñada conforme al número de servicios que se registraron en un ciclo de lactación. Ln $(\mathrm{p} /(1-\mathrm{p}))=\mathrm{b}_{0}+\mathrm{b}_{1}$ BCS1SERV + $\mathrm{b}_{2}$ BCSCONCEP $+\mathrm{b}_{3}$ E1SERV

Donde: Ln $(\mathrm{p} /(1-\mathrm{p}))=$ Probabilidad de éxito para la variable Número de Servicios; $B_{0}=$ Intercepto; BCS1SERV $=$ Valores de la condición corporal de la vaca al primer servicio postparto; $\mathrm{BCSCONCEP}=$ Valores de la condición corporal de la vaca al quedar preñada; E1SERV = Edad de la vaca al primer servicio postparto (meses).

La base de datos digital se analizó con el programa estadístico SAS (StatisticalAnalisysSystem) versión 9.2.

\section{Resultados y discusión}

\section{Producción de leche a los 305 días}

Los valores promedios de las características evaluadas se presentan en el Tabla 1, se muestra a mayor número de lactancia, mayor producción de leche. De acuerdo al Tabla 2, dondeBCSECA, NLACTANCIA y EPARTO fueron asociadas significativamente $(\mathrm{p}<0,01)$ con $\mathrm{P} 305$.

P305 $=10754,80-105,88$ BCSSECA - 612,24BCS1SERV - 167,18BCSCONCEP + 80,73BCSPARTO +440,78 NLACT- 246,945ESTPARTO + 12,83EPARTO

Los parámetros estimados indican que un incremento de un punto en BCSSECA por encima de los parámetros óptimos $(3,0$ a 3,5) están asociados a un menor producción de leche corregida a 305 días (P305), de mantenerse constantes las demás variables en estudio, esto involucraría una pérdida de $105 \mathrm{~kg}$ de leche a los 305 días; para Montiel (2005).
Tabla 2. Parámetros estimados de la regresión lineal múltiple

\begin{tabular}{|c|c|c|c|c|c|}
\hline Variable & GL & Coeficientes & Error & Valor t & $\operatorname{Pr}>F$ \\
\hline Intercepto & 1 & 10754,80 & 820,85 & 6,23 & $<.0,001$ \\
\hline BCS Seca & 1 & $-105,88$ & 96,07 & $-0,29$ & 0,0030 \\
\hline BCS 1er Servicio & 1 & $-612,24$ & 222,60 & 0,31 & 0,7541 \\
\hline BCS Concepción & 1 & $-167,18$ & 166,54 & 0,48 & 0,6282 \\
\hline BCS Parto & 1 & 80,73 & 127,10 & 2,89 & 0,7423 \\
\hline $\mathrm{N}^{\circ}$ de Lactancia & 1 & 440,78 & 295,74 & $-1,49$ & 0,0031 \\
\hline Estación al Parto & 1 & $-246,94$ & 127,41 & 0,15 & 0,8791 \\
\hline Edad al Parto & 1 & 12,83 & 19,86 & 4,22 & $<.0001$ \\
\hline
\end{tabular}

\section{Número de servicios por preñez}

De un total de 797 servicios realizados en 99 vacas entre primíparas y multíparas, resultó 346 inseminaciones exitosas durante el periodo en estudio; se evaluó la probabilidad de un servicio exitoso (vaca preñada $=1$ ) versus uno que no (vaca vacía $=0$ ) a través de un análisis de regresión logística, donde se evalúa la probabilidad de éxito (vaca preñada) de acuerdo al número de servicios que recibió en esa lactancia la vaca. En el Tabla 3, se puede ver la frecuencia por cada servicio en el cual la vaca preñó.

Tabla 3. Frecuencia acumulada de servicios

\begin{tabular}{ccc}
\hline \multicolumn{3}{c}{ Perfil de Respuesta } \\
\hline Valor Ordenado & NSERVICIOS & Frecuencia Total \\
\hline 1 & 1 & 65 \\
2 & 2 & 17 \\
3 & 3 & 8 \\
4 & 4 & 4 \\
5 & 5 & 1 \\
\hline
\end{tabular}

El método de máxima verosimilitud, es el método de estimación de los parámetros para el modelo logístico y nos permite estimar su significancia (Tabla 4); el modelo propuesto para determinar en qué número de servicio se logra una mejor probabilidad de éxito para que una vaca quede preñada, resultó no significativa $(\mathrm{p}>0,05)$ para las variables independientes: BCS1SERV, BCSCONCEP, E1SERV.

Los cambios en la condición corporal durante el secado y parto perjudicarían el retorno a celo de las vacas impactando así en parámetros reproductivos como: días vacíos, intervalo entre partos y número de servicios (Apocada, 2005).

Tabla 1. Promedio de producción de leche, número de servicios y condición corporal en seca, parto, primer servicio y concepción

\begin{tabular}{|c|c|c|c|c|c|c|c|}
\hline $\begin{array}{c}\mathrm{N} \\
\text { Lactancia }\end{array}$ & $\mathrm{N}$ & $\mathrm{P} 305 \pm \mathrm{SD}$ & $\mathrm{NSERV} \pm \mathrm{SD}$ & $\mathrm{BCSSECA} \pm \mathrm{SD}$ & $\mathrm{BCSPARTO} \pm \mathrm{SD}$ & $\mathrm{BCS} 1 \mathrm{SERV} \pm \mathrm{SD}$ & $\begin{array}{c}\mathrm{BCSCONCEP} \pm \\
\text { SD } \\
\end{array}$ \\
\hline 1 & 99 & $8287 \pm 727$ & $1,53 \pm 0,94$ & - & $2,96 \pm 0,28$ & $2,98 \pm 0,12$ & $2,99 \pm 0,10$ \\
\hline 2 & 100 & $8892 \pm 1025$ & $2,28 \pm 1,40$ & $2,59 \pm 0,60$ & $2,75 \pm 0,61$ & $2,44 \pm 0,39$ & $2,46 \pm 0,51$ \\
\hline 3 & 147 & $9844 \pm 1465$ & $2,84 \pm 1,95$ & $2,81 \pm 0,73$ & $2,99 \pm 0,59$ & $2,26 \pm 0,33$ & $2,29 \pm 0,46$ \\
\hline
\end{tabular}

El modelo de regresión lineal múltiple propuesto para la variable P305 explica el 24 por ciento de la variación de la producción de leche, resultó significativo $(\mathrm{p}<0,01)$. 
Tabla 4. Método de máxima verosimilitud para regresión logística

\begin{tabular}{lccccc}
\hline \multicolumn{1}{c}{ Variable } & GL & Estimador & Error & Chi-cuadrado & Pr $>$ Chisq \\
\hline Intercepto 1 & 1 & 31,26420 & 2372,4 & 0,0002 & 0,9895 \\
Intercepto 2 & 1 & 32,37800 & 2372,4 & 0,0002 & 0,9891 \\
Intercepto 3 & 1 & 33,46630 & 2372,4 & 0,0002 & 0,9887 \\
Intercepto 4 & 1 & 35,11490 & 2372,4 & 0,0002 & 0,9882 \\
BCS 1er Servicio & 1 & $-12,53300$ & 790,8 & 0,0003 & 0,9874 \\
BCS Concepción & 1 & 2,44420 & 1,8118 & 1,8200 & 0,1773 \\
$\begin{array}{l}\text { Edad al 1er } \\
\text { Servicio }\end{array}$ & 1 & $-0,01320$ & 0,2183 & 0,0037 & 0,9518 \\
\hline
\end{tabular}

\section{Conclusiones}

La condición corporal en el momento de secado, el número de lactancia y la edad al parto influyeron significativamente $(\mathrm{p}<0,01)$ sobre la producción de leche a los 305 días, de incrementarse un punto de condición corporal durante el secado, por encima del ideal $(3,0$ a 3,5) este impactaría negativamente sobre la producción de leche.

La variable número de servicios no tuvo efectos significativos $(\mathrm{p}>0,05)$ entre está $y$ las variables BCS1SERV, BCSCONCEP Y E1SERV, siendo necesario servir siempre que el animal presente un celo limpio.

\section{Literatura citada}

Apocada, C. 2005. Efecto del Nivel de Producción de Leche sobre el Comportamiento Reproductivo. Asociación Mexicana de Médicos Veterinarios Especialistas en Bovinos, 6: 3-5.

Collantes, C.; Cruz, N.; Vasquez, P. 1999. Análisis TécnicoFinanciero del Establo Lechero "Monte Grande" en Puente Piedra. Tesis Ing. Lima. Universidad Nacional Agraria La Molina, Lima, Perú.

Domecq, J. 1997. Relationship between Body Condition Scores and Milk Yield in a Large Dairy Herd of High Yielding Holstein Cows. Journal Dairy in Science. 80: 101-112.

Garcia, A. 2008. Alimentación de las Vacas Lecheras para Condición Corporal. College of Agriculture and Biological Sciences, 7:1-4. Grigera, J. y Bargo, F. 2005. Evaluación del Estado Corporal en Vacas Lecheras. Producción Bovina de Leche. 1-9.

Hutjens, J. 2007. Señales vacunas, una guía práctica para el manejo de las vacas lecheras. Editorial Hoard'sBookstore, 61-64p.

Lopez, F. 2006. Relación entre Condición Corporal y Eficiencia Reproductiva en Vacas Holstein. Facultad de Ciencias Agropecuarias de la Universidad del Cauca. 4:78-86.

Lucy, M. 2001. Reproductive Loss in High-Producing Dairy Cattle: Where Will It End?. Journal Dairy in Science, 17:1277-1289.

Montiel, F. 2005. Body condition and suckling as factors influencing the duration of postpartum anestrus in cattle: a review. Animal Reproduction Science, 85:1-
26.

Roche, J. 2009. Invited Review: Body condition score and its association with dairy cow productivity, health, and welfare. Journal Dairy in Science, 92:5769-5801.

Ruegg, P. 1995. Body Condition Scores of Holstein Cows on Prince Edward Island, Canada: Relationships with Yield, Reproductive Performance, and Disease. Journal Dairy in Science, 78: 552-564.

SAS Institute Inc. 2008. SAS/STAT 9.2 User's Guide Cary $\mathrm{NC}$ : SAS Institute Inc.

OIE [Organización Internacional para la Salud Animal]. 2008. Report of the OIE ad hoc group on porcine reproductive respiratory syndrome. (Internet) Consultado el 18 de Agosto 2015. 